\title{
On the coefficient bodies of meromorphic univalent functions omitting a disc
}

\author{
by Olli Tammi (Helsinki)
}

\begin{abstract}
Let $S(b)$ be the class of bounded normalized univalent functions and $\Sigma(b)$ the class of normalized univalent meromorphic functions omitting a disc with radius $b$. The close connection between these classes allows shifting the coefficient body information from the former to the latter. The first non-trivial body can be determined in $\Sigma(b)$ as well as the next one in the real subclass $\Sigma_{\mathrm{R}}(b)$.
\end{abstract}

1. Introduction. In characterizing classes of univalent functions, their extremal properties play a central role. Together with certain distortion results the estimation of coefficients offers possibilities of testing the efficiency of the methods available. More general and more demanding is the problem of determining the coefficient bodies, the first of which may allow also nice geometric interpretations. With increasing indices the technical difficulties grow rapidly and the same holds, of course, for possibilities of geometrical interpretation. Thus, usually only the first of these bodies can be thoroughly examined.

Denote by $S$ the basic class of normalized univalent functions $F$, defined in the unit disc $U=\{z|| z \mid<1\}$, i.e.

$$
S=\left\{F \mid F(z)=z+a_{2} z^{2}+\ldots, z \in U\right\} .
$$

The classical treatment concerning the first non-trivial body $\left(a_{3}, a_{2}\right)$, including color photos of its wax models, is given by Schaeffer and Spencer in $[5]$.

$S(b)$ is the class of bounded univalent functions $f$ normalized so that

$$
S(b)=\left\{f\left|f(z)=b\left(z+a_{2} z^{2}+\ldots\right), z \in U,\right| f(z) \mid<1,0<b<1\right\},
$$

with the leading coefficient $b$ constant. In the sense of uniform convergence one can write briefly

$$
S=S(0)
$$

2000 Mathematics Subject Classification: Primary 30C45.

Key words and phrases: univalent functions, coefficient bodies. 
The first attempts to generalize the results of [5] to $S(b)$-functions are those of Charzyński and Janowski [1]. However, the qualitative form of the results leaves the detailed characterization of the body open.

In [3] the missing quantitative information is found by using Grunskytype inequalities in the most economic way, i.e. in so-called optimized form. In [9] and [10] the results are completed to concern the body $\left(a_{3}, a_{2}\right)$ in $S(b)$. Also the next body, $\left(a_{4}, a_{3}, a_{2}\right)$, is studied but only in the real subclass

$$
S_{\mathrm{R}}(b) \subset S(b),
$$

with all the $a_{\nu}$-coefficients real. A further completion is given in [2], where also the complicated elliptic parts of the surface of the body are included.

It seems to be advisable to look for related classes where the above body-program could be repeated. Clearly, this would be possible if the new class is sufficiently close to those mentioned above. The first example of such working connection is the class of non-vanishing univalent functions, which appears to be related to the class $S(b)$. To underline this consistency the class in question is denoted correspondingly:

$$
\left\{\begin{array}{l}
S^{\prime}(B)=\left\{F \mid F(z)=B+A_{1} z+\ldots,\right. \\
\left.\quad z \in U \supset F(U) \not \supset O, 0<B<1, A_{1}>0\right\},
\end{array}\right.
$$

where $S_{\mathrm{R}}^{\prime}(B)$ is the real subclass. Śladkowska [7] gives a close connection between the classes $S_{\mathrm{R}}^{\prime}(B)$ and $S_{\mathrm{R}}(b)$ by aid of which the first coefficient bodies $\left(A_{2}, A_{1}\right)$ and $\left(A_{3}, A_{2}, A_{1}\right)$ of $S_{\mathrm{R}}^{\prime}(B)$ were found in [10].

There exists still one more connection, not yet utilized, where a favorable relationship of the above kind holds. Considering this will be the aim of this paper.

2. Connection between the classes $\Sigma(b)$ and $S(b)$. The basic class for our considerations is the class $\Sigma$ of non-vanishing meromorphic univalent functions $g$ defined by Netanyahu [4]:

$$
\Sigma=\left\{g\left|g(z)=z+\sum_{\nu=0}^{\infty} A_{\nu} z^{-\nu},\right| z \mid>1, g(z) \neq 0\right\} .
$$

In [6] this class is generalized by Siejka to the class $\Sigma_{b}$ of functions $H$ omitting the whole disc $|z| \leq b<1$. For stylistic reasons, let us rewrite $\Sigma_{b}=\Sigma(b)$ :

$$
\Sigma(b)=\left\{H\left|H(z)=z+\sum_{\nu=0}^{\infty} A_{\nu} z^{-\nu},\right| z|>1,| H(z) \mid>b \in\right] 0,1[\} .
$$

In the sense of uniform convergence we may again write

$$
\Sigma=\Sigma(0) \text {. }
$$


In [6] the class $\Sigma(b)$ was found to have a one-to-one relationship with the class $S(b)$, so that

$$
H(z)=\frac{b}{f(1 / z)}, \quad|z|>1 .
$$

This implies for the $A_{\nu^{-}}$and $a_{\nu^{-}}$-coefficients:

$$
\left\{\begin{array}{l}
A_{0}=-a_{2}, \\
A_{1}=-a_{3}+a_{2}^{2}, \\
A_{2}=-a_{4}+2 a_{2} a_{3}-a_{2}^{3},
\end{array}\right.
$$

and conversely

$$
\left\{\begin{array}{l}
a_{2}=-A_{0}, \\
a_{3}=-A_{1}+A_{0}^{2}, \\
a_{4}=-A_{2}+2 A_{0} A_{1}-A_{0}^{3} .
\end{array}\right.
$$

Thus, we are in the desired position trying to shift the optimized information from the coefficient bodies of $S(b)$-functions to the bodies of $\Sigma(b)$-functions.

3. The first non-trivial body $\left(A_{1}, A_{0}\right)$ in $\Sigma(b)$. In [3] and [8] preliminary observations concerning the $S(b)$-body $\left(a_{3}, a_{2}\right)$ can be found. These are completed in [9] by using certain parameters obtained from the slitstructure of the extremal image domain $f(U)$. The geometrical interpretation is achieved by normalizing $a_{2}=\left|a_{2}\right| \geq 0$.

In [9] the formulae are shortened by using the abbreviation $\delta=a_{3}-a_{2}^{2}$. Because this is $-A_{1}$ and $A_{0}=-a_{2}$, the body $\left(a_{3}, a_{2}\right)$ yields directly also the body $\left(A_{1}, A_{0}\right)$. However, to facilitate the use of the results in [9] it is convenient to proceed in terms of $a_{2}$ and $a_{3}$. Let us start by classifying different types of the boundary of $\left(a_{3}, a_{2}\right)$.

Let the boundary type as well as that of the extremal function $f$ and the corresponding extremal domain $f(U)$ us label $m: n$ where $m$ is the number of starting points and $n$ that of end-points of slits in $f(U)$. Because $m \leq n$, in the present case we are dealing with the types $2: 2,1: 2$ and $1: 1$. To connect this with the boundary points take $a_{2}=$ constant and denote the cross-section with the body by $I\left(a_{2}\right)$. In the $a_{3}$-plane the real axis divides this set in two symmetric parts. Let $I_{+}\left(a_{2}\right)$ be the part where $\operatorname{Re} a_{3}>0$. Denote the curved part of the boundary by $\partial I_{+}\left(a_{2}\right)$. We may express the boundary types of this set by using the above symbols, i.e.

$$
\partial I_{+}\left(a_{2}\right)=2: 2, \quad \partial I_{+}\left(a_{2}\right)=2: 2 \cup 1: 2, \quad \ldots
$$


The formulae for the boundary points of $\left(a_{3}, a_{2}\right)$ are in [9]. As an example, take the simplest case.

$\underline{2: 2}$

According to (18), p. 11 of [9] we have for $\partial I\left(a_{2}\right)$

$$
\left|\delta-\delta^{\circ}\right|=R, \quad \delta^{\circ}=\frac{a_{2}^{2}}{2 \ln b}, \quad R=1-b^{2}+\frac{a_{2}^{2}}{2 \ln b} .
$$

The meridian $\partial I\left(a_{2}\right)$ remains to be the whole circle (6) as long as

$$
0 \leq a_{2} \leq 2 b|\ln b|=M_{1} .
$$

For greater values of $a_{2}$ the boundary type $1: 2$ emerges:

$$
\partial I_{+}\left(a_{2}\right)=2: 2 \cup 1: 2 .
$$

Therefore, we turn to the next case.

$\underline{1: 2}$

Applying the optimized Grunsky condition to the rotated function $\tau^{-1} f(\tau z), \tau=e^{i v}$, one ends up with the formulae (35), p. 20 of [9]. The rotation angle and the parameter $\sigma \in[b, 1]$ in (8) have a connection determining the existence condition through the control function $E$ :

$$
\left\{\begin{array}{l}
\sigma \ln \sigma-\sigma+b+\frac{a_{2}}{2}|\cos v|=0, \\
v \in\left[v_{\circ}, \pi\right], \quad v_{\circ}=\overline{\operatorname{arc}} \cos \frac{2 b \ln b}{a_{2}}, \\
E(\sigma)=\sqrt{1-\sigma^{2}}-\sigma \overline{\operatorname{arc}} \cos \sigma-\frac{a_{2}}{2}|\sin v| \geq 0 .
\end{array}\right.
$$

The existence condition $E(\sigma) \geq 0$ holds up to the value $a_{2}=M_{2}$ which is the double zero of

$$
E(\sigma)=\sqrt{1-\sigma^{2}}-\sigma \overline{\operatorname{arc}} \cos \sigma-\sqrt{\frac{a_{2}^{2}}{4}-(\sigma \ln \sigma-\sigma+b)^{2}},
$$

i.e. the root of

$$
E^{\prime}(\sigma)=-\overline{\operatorname{arc}} \cos \sigma+\frac{2 \ln \sigma(\sigma \ln \sigma-\sigma+b)}{\sqrt{a_{2}^{2}-4(\sigma \ln \sigma-\sigma+b)^{2}}}=0 .
$$

This yields for $M_{2}$ :

$$
\left\{\begin{array}{l}
\sqrt{1-\sigma^{2}}-\sigma \overline{\operatorname{arc}} \cos \sigma-\frac{\ln \sigma(\sigma \ln \sigma-\sigma+b)}{\overline{\operatorname{arc} c o s} \sigma}=0 \\
M_{2}=-\frac{2(\sigma \ln \sigma-\sigma+b)}{\overline{\operatorname{arc} c o s} \sigma} \sqrt{\ln ^{2} \sigma+(\overline{\operatorname{arc} c o s} \sigma)^{2}}
\end{array}\right.
$$


If $a_{2}>M_{2}$ the type $1: 1$ arises as a part of the boundary:

$$
\partial I_{+}\left(a_{2}\right)=2: 2 \cup 1: 2 \cup 1: 1 \cup 1: 2 .
$$

Finally, we turn to the boundary-type

\section{$\underline{1: 1}$}

Now we have to consult the formulae (60), p. 45 of [9]. The type of the boundary is again changed if $a_{2} \geq M_{2}$ :

$$
\partial I_{+}\left(a_{2}\right)=2: 2 \cup 1: 1 \cup 1: 2 .
$$

This means that the control function $E(\sigma)$ is no more positive close to the point $\sigma=b$. The critical $a_{2}=M_{3}$ is thus the root of

$$
E(b)=0,
$$

i.e.

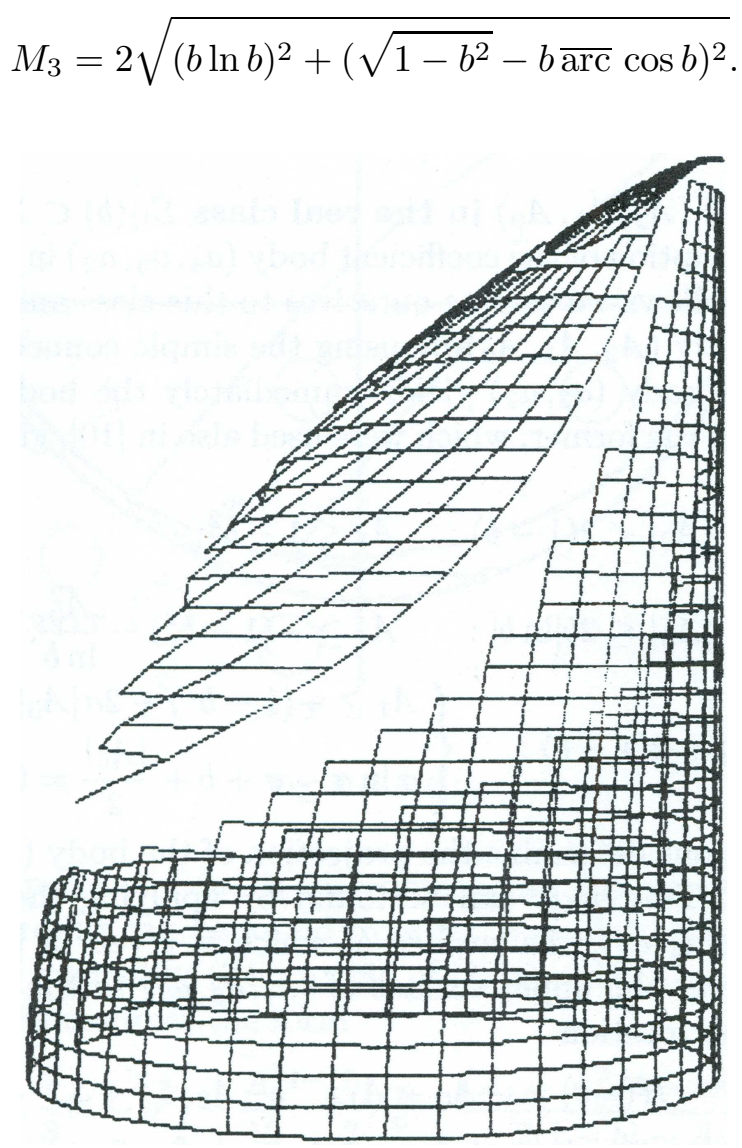

Fig. $1(b=0.1)$ 
Let us collect the boundary restrictions found.

RESUlt. The boundary types of $\left(a_{3}, a_{2}\right)$ are

$$
\begin{array}{ll}
0 \leq a_{2} \leq M_{1}: & \partial I_{+}\left(a_{2}\right)=2: 2, \\
M_{1}<a_{2} \leq M_{2}: & \partial I_{+}\left(a_{2}\right)=2: 2 \cup 1: 2, \\
M_{2}<a_{2}<M_{3}: & \partial I_{+}\left(a_{2}\right)=2: 2 \cup 1: 2 \cup 1: 1 \cup 1: 2, \\
M_{3} \leq a_{2}<2(1-b): & \partial I_{+}\left(a_{2}\right)=2: 2 \cup 1: 1 \cup 1: 2 .
\end{array}
$$

The result remains to hold for $\left(A_{1}, A_{0}\right)$ upon replacing $a_{2}$ by $A_{0} \geq 0$.

Observe that as $b \rightarrow 0$,

$$
M_{3} \rightarrow 2, \quad M_{2} \rightarrow 1.747847823 \ldots
$$

Thus, the last boundary type does not exist in the limit cases $S=S(0)$ and $\Sigma=\Sigma(0)$.

Writing $\delta=-A_{1}, a_{2}=-A_{0}$ in the above-mentioned formulae determining the boundary points, we end up with the body $\left(A_{1}, A_{0}\right)$ in $\Sigma(b)$. In Figure 1 there is a rough picture of this. For clarity, the symmetric parts $1: 2$ and $1: 1$ in the background of the body are left out.

4. The body $\left(A_{2}, A_{1}, A_{0}\right)$ in the real class $\Sigma_{\mathrm{R}}(b) \subset \Sigma(b)$. In [2] there is a detailed description of the coefficient body $\left(a_{4}, a_{3}, a_{2}\right)$ in the real subclass $S_{\mathrm{R}}(b)$. In what follows we confine ourselves to this class and try to shift the results to the body $\left(A_{2}, A_{1}, A_{0}\right)$ by using the simple connections (5).

In $S_{\mathrm{R}}(b)$ the body $\left(a_{3}, a_{2}\right)$ yields immediately the body $\left(A_{1}, A_{0}\right)$. The boundary lines of the former, which were used also in [10], give in the present case:

$$
\begin{aligned}
\left|A_{0}\right|<2(1-b): & A_{1} \leq 1-b^{2} ; \\
\left|A_{0}\right| \leq 2 b|\ln b|: & A_{1} \geq-\left(1-b^{2}\right)-\frac{A_{0}^{2}}{\ln b}, \\
2 b|\ln b| \leq\left|A_{0}\right|<2(1-b): & \left\{\begin{array}{l}
A_{1} \geq-\left(1-b^{2}\right)+2 \sigma\left|A_{0}\right|-2(\sigma-b)^{2}, \\
\sigma \ln \sigma-\sigma+b+\frac{\left|A_{0}\right|}{2}=0, \quad \sigma \in[b, 1] .
\end{array}\right.
\end{aligned}
$$

These conditions determine the projection of the body $\left(A_{2}, A_{1}, A_{0}\right)$ onto the $A_{0} A_{1}$-plane. This body can be found by applying the connection (5) to the body $\left(a_{4}, a_{3}, a_{2}\right)$, the surface of which is given in [2]. The connection (5) transforms the upper surface of $\left(a_{4}, a_{3}, a_{2}\right)$ to the lower surface of $\left(A_{2}, A_{1}, A_{0}\right)$. The rotation

$$
-H(-z)=-A_{0}+A_{1} z^{-1}-A_{2} z^{-2}+\ldots
$$

shows that changing the signs of $A_{0}$ and $A_{2}$ leads finally to the upper surface of the desired body. 
Again, let us characterize the extremal cases by the symbol $m: n, m \leq n$, which in the present case allows the alternatives

$$
3: 3 ; \quad 2: 3, \quad 2: 2 ; \quad 1: 3, \quad 1: 2, \quad 1: 1 .
$$

For the upper surface of the body $\left(a_{4}, a_{3}, a_{2}\right)$ the location of these extremal domains is illustrated in Figure 38, p. 149 of [9]. The corresponding illustration for the upper surface of $\left(A_{2}, A_{1}, A_{0}\right)$ is in Figure 2 below. For brevity, let us preserve the extremal domains in their $S_{\mathrm{R}}(b)$-form. The names of the dividing lines between different extremal types are also preserved in the form $1,2,3 ; 1^{\prime}, 2^{\prime}, 3^{\prime}$. Their equations are direct consequences of those in $S_{\mathrm{R}}(b)$ ([9], pp. 150-153).

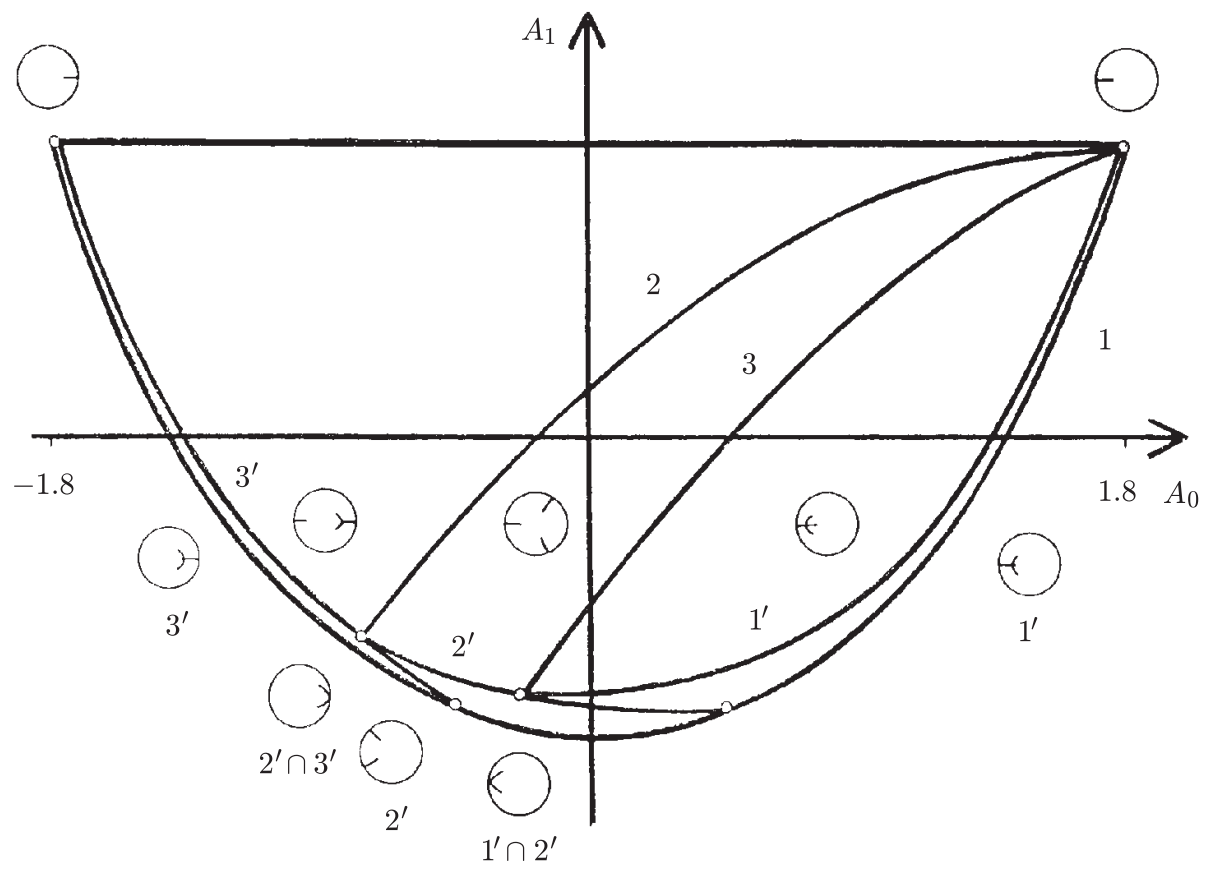

Fig. $2(b=0.1)$

The highest point of $\left(A_{2}, A_{1}, A_{0}\right)$ determines the maximum of $A_{2}$. For $b \in[0.25,1]$ the result can be found from the optimized Grunsky condition, which yields the inequality (24), p. 285 of [8]. For the $A_{\nu}$-coefficients of the upper surface this assumes the form

$$
A_{2} \leq \frac{2}{3}\left(1-b^{3}\right)-\frac{A_{0}^{3}}{12}-\frac{b}{2} A_{0}^{2}-\frac{\left(A_{1}-A_{0}^{2} / 4-b A_{0}\right)^{2}}{2(1-b)-A_{0}} .
$$

The condition holds in the whole $\left(A_{2}, A_{0}\right)$ but is sharp only in the region 
limited by the curves $1^{\prime}, 2^{\prime}$ and 2 . Because

$$
A_{2} \leq \frac{2}{3}\left(1-b^{3}\right)-\frac{A_{0}^{2}}{12}\left(A_{0}+6 b\right)
$$

we see that

$$
A_{2} \leq \frac{2}{3}\left(1-b^{3}\right)
$$

provided

$$
0.25 \leq b<1 \Leftrightarrow-6 b \leq-2(1-b) \leq A_{0} .
$$

Result. In the class $\Sigma_{\mathrm{R}}(b)$,

$$
A_{2} \leq \frac{2}{3}\left(1-b^{3}\right)
$$

for

$$
0.25 \leq b<1 .
$$

Equality holds for symmetric 3-slit stars, i.e. for extremal $S_{\mathrm{R}}(b)$-functions $f$ for which $a_{2}=a_{3}=0$ and

$$
\frac{f^{3}}{\left(1-f^{3}\right)^{2}}=b^{3} \frac{z^{3}}{\left(1-z^{3}\right)^{2}} .
$$

For the remaining interval

$$
0<b<0.25
$$

we have to content ourselves with the numerical checking of the upper surface of the coefficient body.

The formulae determining the algebraic parts of $\left(a_{4}, a_{3}, a_{2}\right)$ are (22), p. 133, and (7), p. 156, in [9]. These results are also collected in the conditions (1)-(3) of [2]. Through (5) their contents are transformed to concern the upper algebraic parts of the body $\left(A_{2}, A_{1}, A_{0}\right)$.

$\underline{2: 3}$

$$
\left\{\begin{aligned}
A_{0}= & 2(1-b)+4\left(\sigma_{1}-\sigma_{2}\right)-\frac{8}{3}\left(1-\sigma_{2}^{3 / 2}\right), \\
A_{1}= & 1-b^{2}-\frac{16}{9}\left(1-\sigma_{2}^{3 / 2}\right)^{2}, \\
A_{2}= & b^{2}\left[2(1-b)+4\left(\sigma_{1}-\sigma_{2}\right)-\frac{8}{3}\left(1-\sigma_{2}^{3 / 2}\right)\right] \\
& \quad+\frac{32}{9}\left(1-\sigma_{2}^{3 / 2}\right)^{2}-\left(\frac{4}{3}\right)^{4}\left(1-\sigma_{2}^{3 / 2}\right)^{3}, \\
b \leq & \sigma_{1} \leq \sigma_{2} \leq 1 .
\end{aligned}\right.
$$


Clearly, $A_{2}$ is maximized if $\sigma_{1}=\sigma_{2}$, i.e. on the boundary arc 2. Thus, it remains to maximize

$$
\left\{\begin{array}{l}
A_{2}=2(1-b) b^{2}+G(t) \\
G(t)=-2 b^{2} t+2 t^{2}-\frac{4}{3} t^{3} \\
t=\frac{4}{3}\left(1-\sigma_{2}^{3 / 2}\right) \in\left[0, \frac{4}{3}\left(1-b^{3 / 2}\right)\right]
\end{array}\right.
$$

which on the interval (13) yields

$$
\max A_{2}=2(1-b) b^{2}+G\left(\frac{1+\sqrt{1-2 b^{2}}}{2}\right)<\frac{2}{3}\left(1-b^{3}\right) .
$$

\section{$\underline{3: 3 \cup 1: 3}$}

The equations for the present part of the upper surface assume the form

$$
\left\{\begin{array}{l}
A_{0}=\frac{1}{3}(8 \sigma-6 b-2)+\frac{2}{3}\left(1-\sigma^{-1 / 2}\right) u \\
A_{1}=\frac{1}{9}\left(16 \sigma^{2}-9 b^{2}-7\right)+\frac{8}{9}\left(1-\sigma^{1 / 2}\right) u-\frac{1}{9}\left(1-\sigma^{-1}\right) u^{2} \\
A_{2}=\frac{2}{3}\left(1-b^{3}\right)+\frac{A_{0}^{3}}{12}-\frac{b}{2} A_{0}^{2}+\frac{u-1}{3}\left(\frac{A_{0}^{2}}{4}-b A_{0}-A_{1}\right), \\
\sigma \in[b, 1], u \in\left[-8 \sigma^{3 / 2}, 4 \sigma^{3 / 2}\right] .
\end{array}\right.
$$

By using the notations

$E_{0}=16 \sigma^{2}+8 \sigma^{1 / 2}-9 b^{2}, \quad E_{1}=1-8 \sigma^{1 / 2}+\sigma^{-1 / 2}, \quad E_{2}=\sigma^{-1}-2 \sigma^{-1 / 2}-2$, we can write the local extremal conditions in the form

$$
\left\{\begin{aligned}
\frac{\partial A_{2}}{\partial u}= & \frac{2}{27}\left(1-\sigma^{-1 / 2}\right)\left(E_{0}+2 E_{1} u+E_{2} u^{2}\right)=0 \\
\frac{\partial A_{2}}{\partial \sigma}= & -\left[\left(\frac{A_{0}}{2}\right)^{2}+\left(\frac{u-1}{3}-2 b\right) \frac{A_{0}}{2}+\frac{u-1}{3} \cdot b\right] \frac{\partial A_{0}}{\partial \sigma} \\
& +\frac{u-1}{3} \cdot \frac{\partial A_{1}}{\partial \sigma}=0 \\
\frac{\partial A_{0}}{\partial \sigma}= & -\frac{1}{3}\left(8+\sigma^{-3 / 2} u\right) \\
\frac{\partial A_{1}}{\partial \sigma}= & \frac{4}{9}\left(8-\sigma^{-1 / 2} u\right)
\end{aligned}\right.
$$

In Table 1 there are examples of solutions of the system (16) showing that the previous candidate (12) preserves its role also in the present case. 
Table 1

\begin{tabular}{|c|c|c|c|c|}
$b$ & $\sigma$ & $A_{0}$ & $A_{1}$ & $A_{2}$ \\
\hline 0.05 & 0.422235729 & 0 & 0 & 0.666583333 \\
0.10 & 0.448359266 & 0 & 0 & 0.666000000 \\
0.15 & 0.475172339 & 0 & 0 & 0.664416666 \\
0.20 & 0.502627925 & 0 & 0 & 0.661333333 \\
0.25 & 0.530681002 & 0 & 0 & 0.656250000
\end{tabular}

Elliptic cases $1: 2$ and $2: 2$

There remain the elliptic cases which for $\left(a_{4}, a_{3}, a_{2}\right)$ are analyzed in [2], Chapters 3 and 4 . The $2: 2$-solution is based on numerical integration of differential equations (7), p. 6 in [2]. The $1: 2$-cases can be found by superimposing a proper limiting $2: 2$-function with correctly chosen radialslit mapping. The connection (5) yields finally the elliptic points of the upper surface of the body $\left(A_{2}, A_{1}, A_{0}\right)$. In Figure 2 the elliptic points belong to the region restricted by the arcs $1,1^{\prime}, 2^{\prime}, 3^{\prime}$.

\section{Table 2}

\begin{tabular}{|c|c|c|c|}
$R$ & $A_{0}$ & $A_{1}$ & $A_{2}$ \\
\hline-2 & -1.384 & -0.016 & 0.653 \\
-8 & -1.372 & -0.038 & 0.652 \\
-32 & -1.350 & -0.076 & 0.647 \\
-128 & -1.332 & -0.107 & 0.624 \\
-512 & -1.322 & -0.126 & 0.375 \\
$S=13.778495$ \\
$b=0.1$
\end{tabular}

An example of elliptic boundary points belonging to the type $1: 2$, with right-hand radial slit, is given in Table 2. It belongs to a curve starting from the maximal point on the arc $3^{\prime} . S$ and $R$ are parameters fixing the curve.

Checking the elliptic point shows that the maximum occurs on the boundary arc $3^{\prime}$. This suggests strongly that also for the interval (13) the above sharp upper bound (12) remains to hold.

The formulae (14) and (15) together with the elliptic information in [2] allow visualizing the coefficient body $\left(A_{2}, A_{1}, A_{0}\right)$. This is done in Figure 3 by aid of proper nets. To avoid too much overlapping the algebraic and elliptic parts of the surface are drawn separately. Observe that the upper and lower surface are obtainable from each other by aid of two rotations. 


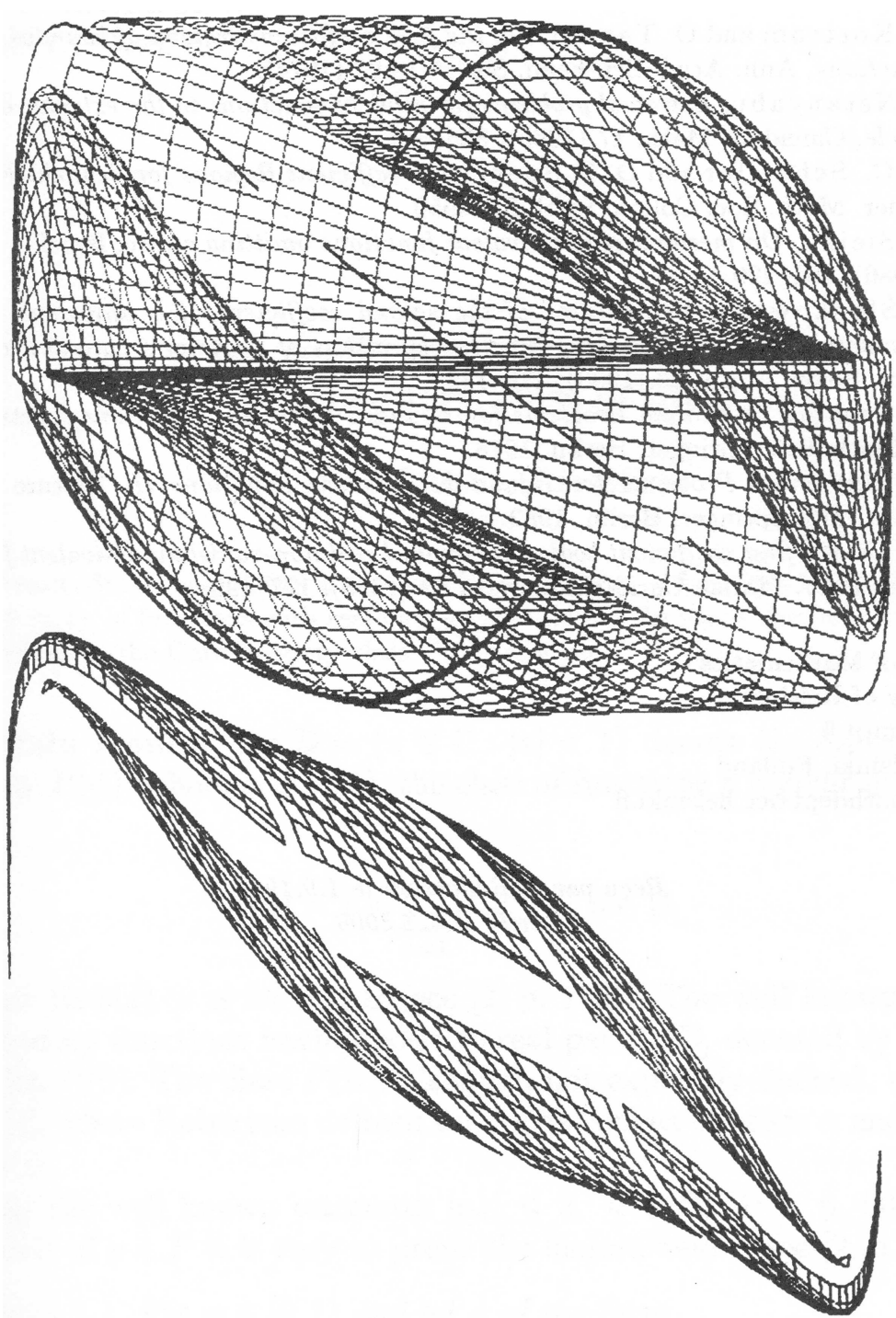

Fig. $3(b=0.1)$

\section{References}

[1] Z. Charzyński and W. Janowski, Domaine de variation des coefficients $A_{2}$ et $A_{3}$ des functions univalentes et bornées, Bull. Soc. Sci. Lettres Łódź 10 (1959), no. 5 .

[2] O. Jokinen and O. Tammi, On determining the points of the second coefficient body $\left(a_{4}, a_{3}, a_{2}\right)$ for bounded real univalent functions, Kodai Math. J. 17 (1994), $82-100$. 
[3] R. Kortram and O. Tammi, On the first coefficient regions of bounded univalent functions, Ann. Acad. Sci. Fenn. Ser. A I 592 (1974).

[4] E. Netanyahu, Extremal problems for schlicht functions in the exterior of the unit circle, Canad. J. Math. 17 (1965), 335-341.

[5] A. C. Schaeffer and D. C. Spencer, Coefficient Regions for Schlicht Functions, Amer. Math. Soc. Colloq. Publ. 35, 1950.

[6] H. Siejka, On meromorphic univalent functions omitting a disc, Israel J. Math. 54 (1986), 291-299.

[7] J. Sladkowska, Estimations of the second coefficient of a univalent, bounded, symmetric and non-vanishing function by means of Loewner's parametric method, Ann. Polon. Math. 68 (1998), 119-123.

[8] O. Tammi, Extremum Problems for Bounded Univalent Functions, Lecture Notes in Math. 646, Springer, Berlin, 1978.

[9] —, Extremum Problems for Bounded Univalent Functions II, Lecture Notes in Math. 913, Springer, Berlin, 1982.

[10] - On the first coefficient bodies of bounded real non-vanishing univalent functions, Ann. Univ. Mariae Curie-Skłodowska 52 (1998), 177-190.

Institute of Mathematics

University of Helsinki

Yliopistokatu 5

00014 Helsinki, Finland

E-mail: mathdept@cc.helsinki.fi 